\title{
Carbon-rich AGB stars in our Galaxy and nearby galaxies as possible sources of PAHs
}

\author{
M. Matsuura ${ }^{1}$, G.C. Sloan ${ }^{2}$, J. Bernard-Salas ${ }^{2}$, A. A. Zijlstra ${ }^{3}$, \\ P. R. Wood ${ }^{4}$, P. A. Whitelock ${ }^{5,6,7}$, J. W. Menzies ${ }^{5}$, M. Feast $^{6}$, \\ E. Lagadec ${ }^{3}$, M. A. T. Groenewegen ${ }^{8}$, \\ M. R. Cioni ${ }^{9}$, J. Th. van Loon ${ }^{10}$, and G. Harris ${ }^{11}$
}

${ }^{1}$ National Astronomical Observatory of Japan, Osawa 2-21-1, Mitaka, Tokyo 181-8588, Japan email: m.matsuura@nao.ac.jp

${ }^{2}$ Astronomy Department, Cornell University, 610 Space Sciences Building, Ithaca, NY 14853-6801, USA

${ }^{3}$ Jodrell Bank Centre for Astrophysics, School of Physics and Astronomy, The University of Manchester, Oxford Street, Manchester M13 9PL, UK

${ }^{4}$ Research School of Astronomy \& Astrophysics, Mount Stromlo Observatory, Australian National University, Cotter Road, Weston ACT 2611, Australia

${ }^{5}$ South African Astronomical Observatory, P.O. Box 9, 7935 Observatory, South Africa

${ }^{6}$ Astronomy Department, University of Cape Town, 7701 Rondebosch, South Africa

${ }^{7}$ NASSP, Department of Mathematics and Applied Mathematics, University of Cape Town, 7701 Rondebosch, South Africa

${ }^{8}$ Instituut voor Sterrenkunde, KU Leuven, Celestijnenlaan 200B, 3001 Leuven, Belgium

${ }^{9}$ Centre for Astrophysics Research, University of Hertfordshire, Hatfield AL10 9AB, UK

${ }^{10}$ Astrophysics Group, School of Physical and Geographical Sciences, Keele University, Staffordshire ST5 5BG, UK

${ }^{11}$ Department of Physics and Astronomy, University College London, Gower Street, London WC1E 6BT, UK

\begin{abstract}
We have obtained infrared spectra of carbon-rich AGB stars in three nearby galaxies the Large and Small Magellanic Clouds, and the Fornax dwarf spheroidal galaxy. Our primary aim is to investigate gas compositions and mass-loss rate of these stars as a function of metallicity, by comparing AGB stars in several galaxies with different metallicities. $\mathrm{C}_{2} \mathrm{H}_{2}$ are detectable from AGB stars, and possibly PAHs are subsequently formed from $\mathrm{C}_{2} \mathrm{H}_{2}$. Thus, it is worth investigating chemical processes at low metallicity. These stars were observed using the Infrared Spectrometer (IRS) onboard the Spitzer Space Telescope which covers 5-35 $\mu \mathrm{m}$ region, and the Infrared Spectrometer And Array Camera (ISAAC) on the Very Large Telescope which covers the 2.9-4.1 $\mu$ m region. $\mathrm{HCN}, \mathrm{CH}$ and $\mathrm{C}_{2} \mathrm{H}_{2}$ molecular bands, as well as $\mathrm{SiC}$ and $\mathrm{MgS}$ dust features are identified in the spectra. The equivalent width of $\mathrm{C}_{2} \mathrm{H}_{2}$ molecular bands is larger at lower metallicity, thus PAHs might be abundant in AGB stars at low metallicity. We find no evidence that mass-loss rates depend on metallicity. Chemistry of carbon stars is affected by carbon production during the AGB phase rather than the metallicities. We argue that lower detection rate of PAHs from the interstellar medium of lower metal galaxies is caused by destruction of PAHs in the ISM by stronger UV radiation field.
\end{abstract}

Keywords. Stars: AGB and post-AGB, ISM: molecules, stars: late-type, (stars:) circumstellar matter, (galaxies:) Magellanic Clouds, (galaxies:) Local Group 


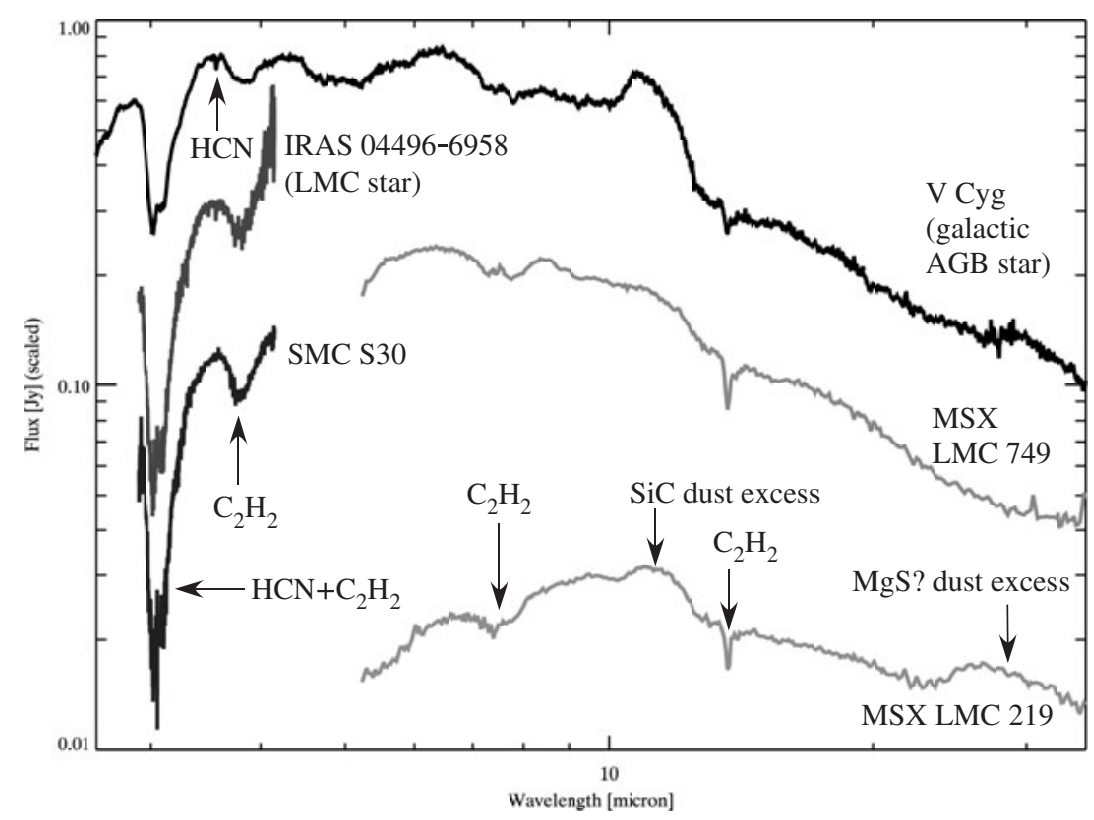

Figure 1. Infrared spectra of Galactic, LMC and SMC carbon-rich AGB stars

\section{Introduction}

Most polycyclic aromatic hydrocarbons (PAHs) are probably formed in carbon-rich asymptotic giant branch (AGB) stars (Allamandola et al. 1989). However, PAHs are undetected from AGB stars, because of the lack of UV radiation from these low temperature stars (effective temperature of 2000-3000 K). Instead, $\mathrm{C}_{2} \mathrm{H}_{2}$, which is a parent molecule to form PAHs (Allamandola et al. 1989), is detectable in AGB stars. This motivates us to study $\mathrm{C}_{2} \mathrm{H}_{2}$ in AGB stars.

AGB stars distribute their chemical products through mass loss process. The mass loss from AGB stars is intense; approximately $50-80 \%$ of the stellar mass is lost during the AGB phase for Galactic stars. Understanding this process and the composition of the gas lost from AGB stars is important for understanding both stellar and galactic evolution. In particular, carbon-rich molecules are primarily formed in carbon-rich AGB stars. Theoretical work has suggested that AGB mass-loss rates depend on metallicity (Bowen \& Willson 1991, Willson 2006), because the stellar wind is triggered by radiation pressure on dust grains and the dust is made up of astronomical metals. Therefore a study of mass-loss rate of extra-galactic AGB stars is vital, because it allows to study chemical enrichment process at low metallicity.

\section{Observations and results}

We have obtained infrared spectra, using the Very Large Telescope (VLT) and the Spitzer Space Telescope (SST). This paper summarises our series of studies (Matsuura et al. 2002, 2005, 2006, 2007, Sloan et al. 2006, van Loon et al. 2006, Zijlstra et al. 2006, Lagadec et al. 2007, 2008) of the 3-35 $\mu \mathrm{m}$ spectra of extragalactic carbon stars. Our targets are located in three nearby galaxies, namely the Large Magellanic Cloud (LMC; $[\mathrm{Fe} / \mathrm{H}] \sim-0.3)$, the Small Magellanic Cloud $(\mathrm{SMC} ;[\mathrm{Fe} / \mathrm{H}] \sim-0.6)$, and the Fornax dSph galaxy $([\mathrm{Fe} / \mathrm{H}] \sim-1.0)$.

Figure 1 shows the spectrum of a star in our Galaxy obtained by the Infrared Space Observatory (ISO) and spectra of LMC and SMC stars observed with VLT and Spitzer. 


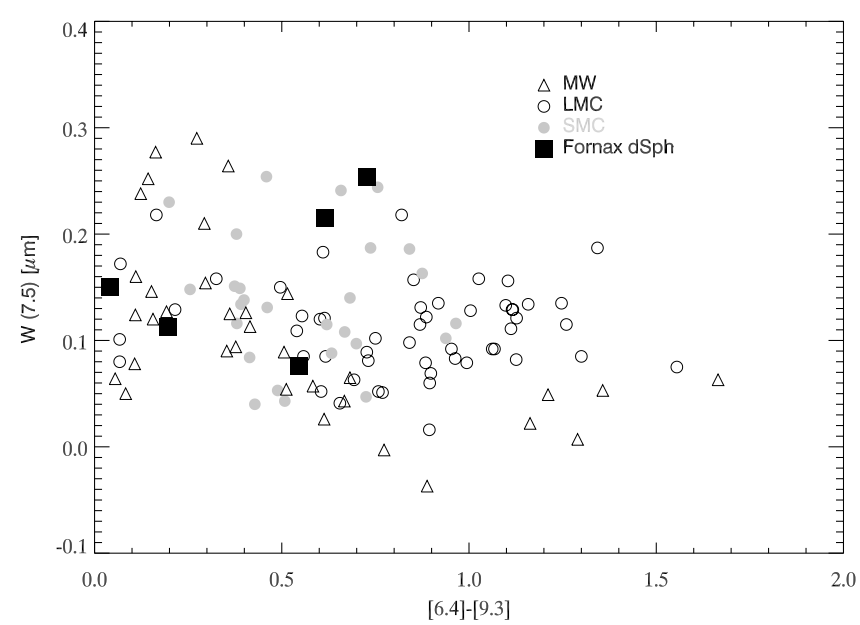

Figure 2. The equivalent width of $7.5 \mu \mathrm{m} \mathrm{C}_{2} \mathrm{H}_{2}$ as a function of infrared colour [6.4]- [9.3]. Symbols show the host galaxies, MW representing the Milky Way.

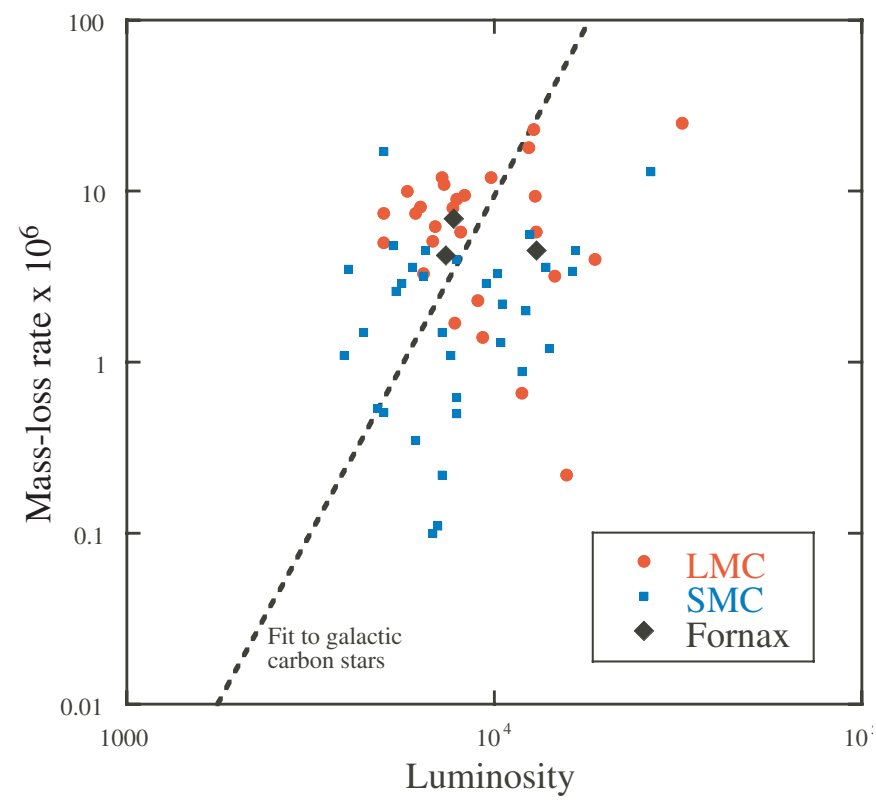

Figure 3. Mass-loss rate $\left(\mathrm{M}_{\odot} \mathrm{yr}^{-1}\right)$ as a function of luminosity $\left(\mathrm{L}_{\odot}\right)$ for Formax sample (Matsuura et al. 2007) and for the LMC and SMC samples (Groenewegen et al. 2007). Fornax stars show high mass-loss rates for their luminosities. The dotted line is the fit to the luminosity vs mass-loss rate relation for Galactic carbon stars (Groenewegen et al. 1998).

Major molecular bands and dust excess are indicated in the figure. $\mathrm{C}_{2} \mathrm{H}_{2}$ bands are found at $3.8,7$, and $13 \mu \mathrm{m}$. The $3.1 \mu \mathrm{m}$ absorption is due to $\mathrm{HCN}$ and $\mathrm{C}_{2} \mathrm{H}_{2}$. There are two dust features found, $\mathrm{SiC}$ at $11.3 \mu \mathrm{m}$ and $\mathrm{MgS}$ at $\sim 30 \mu \mathrm{m}$.

First we measured the equivalent widths of the molecular bands, so as to evaluate the metallicity dependence of these features. The equivalent width of the $\mathrm{C}_{2} \mathrm{H}_{2}$ molecular bands are measured following the method of Zijlstra et al. (2006). Figure 2 shows the 
$7.5 \mu \mathrm{m} \mathrm{C}_{2} \mathrm{H}_{2}$ equivalent width as a function of infrared colour [6.4]-[9.3]. The [6.4] and [9.3] values are calculated from the Spitzer/IRS spectra, and the definition of these magnitudes is also given by Zijlstra et al. (2006). Within the $0.6<[6.4]-[9.3]<1.0$ range, a high value of $\mathrm{W}(7.5)$, with respect to infrared colour, is found for stars in Fornax and the SMC while a low $\mathrm{W}(7.5)$ is found in our Galaxy. This infrared colour measures effective temperature of stars for blue stars, but also circumstellar dust excess for red stars. $\mathrm{C}_{2} \mathrm{H}_{2}$ equivalent width increases towards lower metallicity.

Figure 3 shows the derived mass-loss rate as a function of luminosity. This is also confirmed by the further LMC study of Sloan et al. (2008). Low metallicity dependence of mass-loss rates is found for carbon-rich stars. The stars in Fornax dSph galaxy are at the upper end of the SMC mass-loss rates at a given luminosity. LMC stars appear to reach a higher mass-loss rate than the SMC and Fornax stars at a given luminosity.

\section{Discussion}

We found that $\mathrm{C}_{2} \mathrm{H}_{2}$ is more abundant at low metallicity. $\mathrm{C}_{2} \mathrm{H}_{2}$ formation relies on excess carbon atoms after all oxygen atoms are locked into carbon monoxide. Thus the higher abundance of $\mathrm{C}_{2} \mathrm{H}_{2}$ at lower metallicity is due to the higher $\mathrm{C} / \mathrm{O}$ ratio (Matsuura et al. 2005) caused by carbon synthesised in AGB stars. $\mathrm{C}_{2} \mathrm{H}_{2}$ is thought to be a parent molecule in the formation of PAHs, as indicated by chemical models (Allamandola et al. 1989). It is still unknown whether PAHs are formed during the AGB phase or afterwards. If PAHs are indeed formed during the AGB phase, an over-abundance of $\mathrm{C}_{2} \mathrm{H}_{2}$ in a low metal environment will affect the growth of these important molecules. This implies that weaker PAH bands from the interstellar medium of lower metal galaxies could be caused by destruction of strong UV radiation fields at lower metallicities.

\section{References}

Allamandola, L. J., Tielens, G. G. M., \& Barker, J. R. 1989, ApJS, 71, 733

Bowen, G. H. \& Willson, L. A. 1991, ApJ (Letter), 375, L53

Groenewegen, M. A. T., Whitelock, P. A., Smith, C. H., \& Kerschbaum, F. 1998, MNRAS, 293, 18

Groenewegen, M. A. T., Wood, P. R., Sloan, G. C., et al. 2007, MNRAS, 376, 313

Lagadec, E., Zijlstra, A. A., Matsuura, M., Menzies, J. W., van Loon, J. Th., \& Whitelock, P. A. 2008, MNRAS, 383, 399

Lagadec, E., Zijlstra, A. A., Sloan, G. C., et al. 2007, MNRAS, 376, 1270

Matsuura, M., Wood, P. R., Sloan, G. C., et al. 2006, MNRAS, 371, 415

Matsuura, M., Zijlstra, A. A., Bernard-Salas, J., et al. 2007, MNRAS, 382, 1889

Matsuura, M., Zijlstra, A. A., van Loon, J. Th., et al. 2002, ApJ (Letter), 580, L133

Matsuura, M., Zijlstra, A. A., van Loon, J. Th., et al. 2005, A\&A, 434, 691

Sloan, G. C., Kraemer, K. E., Matsuura, M., et al. 2006, ApJ 645, 1118

Sloan, G. C., Kraemer, K. E., Wood, P. R., et al. 2008, ApJ, in press, astro-ph/0807.2998

van Loon, J. Th., Marshall, J. R., Cohen, M., et al. 2006, A\&A, 447, 971

Willson, L. A. 2006, in: Letizia Stanghellini, J. R. Walsh, and N.G. Douglas (eds.), Planetary nebulae beyond the Milky Way, Proceedings of the ESO workshop (Berlin: Springer), p. 99

Zijlstra, A. A., Matsuura, M., van Loon, J. Th., et al. 2006, MNRAS, 370, 1961 